\title{
A abordagem do bruxismo em paciente infantil: relato de caso
}

\author{
The brussels approach in child patient: case report
}

El enfoque de bruselas en el paciente infantil: informe de caso

Paulo Victor Oliveira Paiva ${ }^{1 *}$, Ivana Letícia Teixeira Morais ${ }^{1}$, Danielly Costa Sábio ${ }^{1}$, Henrique Fayad Pinheiro ${ }^{1}$, Dóris Kós Burlamaqui de Miranda ${ }^{1}$, Jesus Maués Pinheiro Júnior ${ }^{1}$.

\section{RESUMO}

Objetivo: Apresentar, por meio de um relato de caso, os fatores etiológicos, as consequências e os possíveis tratamentos para o bruxismo. Detalhamentos de Caso: Paciente com três anos e seis meses, sexo feminino, no período da dentadura decídua, compareceu à clínica odontológica do Cesupa apresentando desgaste significativo nos dentes anteriores e posteriores superiores e inferiores. Os pais relataram que a criança produzia ruídos durante o sono, essa atividade aumentava também quando a criança era submetida a momentos de maior ansiedade. Na avaliação intrabucal, verificou-se que os dentes estavam desgastados, porém com maior severidade para os incisivos superiores e inferiores. No contexto geral, a paciente apresentava características de oclusão próximas da normalidade para esta fase do desenvolvimento da oclusão. Considerações finais: O estudo da origem e das características clínicas do bruxismo na infância é de grande importância para que o diagnóstico seja o mais rápido possivel, permitindo que odontopediatras, pediatras e psicólogos possam determinar um tratamento multidisciplinar e que favoreça o desenvolvimento integral da criança para a promoção de saúde e bem-estar individual. Portanto, o cirurgião dentista deve estar sempre atento aos sinais e sintomas que pacientes possam vir a apresentar em seu consultório.

Palavras-chave: Bruxismo, Odontopediatria, Reabilitação bucal.

\begin{abstract}
Objective: To present, through a case report, the etiological factors, the consequences and possible treatments for bruxism. Case Details: Female patient, three years and six months old, in the period of primary dentition, attended the dental clinic of Cesupa showing significant wear on the upper and lower anterior and posterior teeth. Parents reported that the child produced noise during sleep, this activity also increased when the child was subjected to moments of greater anxiety. In the intraoral evaluation, it was found that the teeth were worn, but with greater severity for the upper and lower incisors. In the general context, the patient had occlusion characteristics close to normal for this stage of the development of the occlusion. Final considerations: The study of the origin and clinical characteristics of bruxism in childhood is of great importance so that the diagnosis is as fast as possible, allowing pediatric dentists, pediatricians and psychologists to determine a multidisciplinary treatment and that favors the integral development of the child for the promotion of individual health and well-being. Therefore, the dental surgeon must always be attentive to the signs and symptoms that patients may have in their office.
\end{abstract}

Keywords: Bruxism, Pediatric dentistry, Oral rehabilitation.

${ }^{1}$ Centro Universitário do Estado do Pará (CESUPA), Belém - PA.

*E-mail: paivapaulovictor@yahoo.com.br

SUBMETIDO EM: 7/2020

ACEITO EM: 8/2020

PUBLICADO EM: 11/2020 


\section{RESUMEN}

Objetivo: Presentar, a través de un caso clínico, los factores etiológicos, las consecuencias y los posibles tratamientos para el bruxismo. Detalles del Caso: Paciente de tres años y seis meses de edad, en el período de dentición primaria, asistió a la clínica dental de Cesupa mostrando un desgaste significativo en los dientes anteriores y posteriores superiores e inferiores. Los padres informaron que el niño produjo ruido durante el sueño, esta actividad también aumentó cuando el niño estuvo sujeto a momentos de mayor ansiedad. En la evaluación intraoral, se encontró que los dientes estaban desgastados, pero con mayor severidad para los incisivos superiores e inferiores. En el contexto general, el paciente tenía características de oclusión cercanas a lo normal para esta etapa del desarrollo de la oclusión. Consideraciones finales El estudio del origen y características clínicas del bruxismo en la infancia es de gran importancia para que el diagnóstico sea lo más rápido posible, permitiendo a los odontopediatras, pediatras y psicólogos determinar un tratamiento multidisciplinario y que favorezca el desarrollo integral del niño para la promoción de salud y bienestar individual. Por ello, el cirujano dentista debe estar siempre atento a los signos y síntomas que puedan tener los pacientes en su consultorio.

Palabras clave: Bruxismo, Odontología pediátrica, Rehabilitación oral.

\section{INTRODUÇÃO}

O termo bruxismo vem do grego "bruchein" e tem como significado apertamento, friç̧ão ou atrito dos dentes. Trata-se de uma atividade involuntária e repetitiva, caracterizada por ser um transtorno parafuncional que afeta a musculatura da mastigação. Pode ser classificada como bruxismo excêntrico, que é o hábito de ranger os dentes, ou como bruxismo cêntrico, que é o habito de apertar os dentes (GONÇALVES LPV, 2010).

O bruxismo ainda não possui uma etiologia concreta, sendo esta multifatorial, e considera-se que as causas principais que o determinam são: locais, psicológicas, sistêmicas e genéticas. Gera traumas, hábitos parafuncionais, tensões e iatrogenias, sobrepondo-se à tolerância fisiológica de cada indivíduo, que é influenciado por fatores locais, como estabilidade articular, determinados pela oclusão, relações articulares anormais ou ambas, e também por fatores genéticos, sexo e dieta (REAL A, 2018).

Rios LT (2018), ressalta o fato de que a etiologia do bruxismo não está completamente esclarecida, uma vez que há controvérsias nas pesquisas ao se discutir se fatores psicológicos podem influenciar no desencadeamento e manutenção dessa desordem (RIOS LT, 2018).

Os hábitos parafuncionais são danosos e têm grande importância no desenvolvimento da Disfunção Temporomandibular (DTM'S), pois os músculos da mastigação geralmente estão acarretando fadiga muscular, o que resulta em desgaste anormal e em amolecimento pelo ato de o paciente ranger e apertar os dentes, sendo este o sinal clínico mais evidente dessa parafunção.

A sensação dolorosa nos tecidos musculares é indicativa de danos nos tecidos pela alteração do seu metabolismo histológico, uma vez que fibras musculares lesadas estão associadas com processos inflamatórios reparadores, o que explica a sensação dolorosa nessas situações (PACHECO MCT, 2015).

O bruxismo do sono (BS) é uma condição frequente em adolescentes, e os fatores associados à sua maior prevalência foram: sexo masculino, ronco e dificuldades para dormir (SOUZA KM, 2010). O diagnóstico do bruxismo ainda é um desafio para o cirurgião-dentista.

Devido a sua natureza multifatorial, torna-se importante estabelecer o diagnóstico com base nos possíveis fatores etiológicos e não apenas nos sinais clínicos. Portanto, existe um sistema de classificação de pacientes segundo suas necessidades de diagnóstico, sendo estes categorizados em três níveis diferentes, como: "possível", "provável" e "definitivo". 
O nível "possível" baseia-se apenas em autorrelatos por meio de questionários ou métodos anamnésicos; o nível "provável" inclui o mesmo critério do anterior e acrescenta sinais durante o exame clínico; o nível "definitivo", para além dos autorrelatos e sinais clínicos, compreende também o exame polissonográfico que consiste em monitorar as atividades do paciente durante o sono, verificando atividade cerebral, cardíaca, muscular e respiratória (CASTRILLON EE, 2016).

Restrepo CC et al. (2011), exaltam o fato de que a ocorrência do bruxismo durante a infância não é considerada uma patologia até 0 momento em que danos nas estruturas associadas ao sistema estomatognático (músculos, dentes, mucosas e articulação temporomandibular) sejam observados. A identificação de fatores psicossociais, como ansiedade, estresse e características de personalidade, deve ser considerada para um melhor entendimento no desenvolvimento do bruxismo.

Cada paciente deve ser avaliado e tratado individualmente conforme fatores predisponentes, para evitar possíveis complicações, pois não há tratamentos específicos para o bruxismo. Sendo assim, o bruxismo é considerado uma ocorrência frequente na infância, e o diagnóstico suscita questionamentos aos pais e por parte dos clínicos para uma avaliação da qualidade de vida que esta condição parafuncional traz para a criança e seus familiares (MACHADO E, 2011).

O tratamento do bruxismo consiste em um trabalho multidisciplinar que abrange a odontologia, a medicina e a psicologia. No tratamento odontológico, a intervenção clínica deve ser voltada para a proteção dos dentes, aliviando dores faciais e temporais, reduzindo o ranger e melhorando a qualidade do sono e, assim, a qualidade de vida do paciente.

A placa de mordida é a forma de tratamento mais indicada na odontologia: trata-se de um dispositivo interoclusal removível, que abrange todos os dentes de um dos arcos, geralmente o superior, que se ajusta nas incisais e oclusais dos dentes, criando contato oclusal prévio com os dentes do arco oposto (GIMENES MCM, 2008).

Haja vista a complexidade que o bruxismo apresenta na sua identificação e abordagem de tratamento em paciente infantil, o presente artigo tem como objetivo apresentar, por meio de um relato de caso, os fatores etiológicos, as consequências e os possíveis tratamentos para o bruxismo.

\section{DETALHAMENTOS DE CASO}

Paciente com três anos e seis meses, sexo feminino, no período da dentadura decídua, apresentava desgaste significativo nos dentes anteriores e posteriores superiores e inferiores. Os pais relataram que a criança produzia ruídos durante o sono, o que, segundo eles, era provocado pelo ranger dos dentes desde aproximadamente os dois anos de idade.

Informaram ainda que essa atividade aumentava também quando a criança era submetida a momentos de maior ansiedade. A família relatou como queixa principal "desgastes nos dentes da frente", com a preocupação de que esse quadro evoluísse para um desgaste ainda maior.

Ao exame clínico, verificou-se na análise facial em norma frontal e lateral que a paciente apresentava um padrão I de crescimento facial, com tipologia braquifacial, evidenciando simetria e proporção normais (Figuras 1a e 1b) e na análise do sorriso, diagnosticou-se ainda pouca exposição de incisivos superiores, denotandose ainda o visível desgaste nas regiões incisais dos incisivos superiores.

$\mathrm{Na}$ avaliação intrabucal, verificou-se que os dentes estavam desgastados, porém com maior severidade para os incisivos superiores e inferiores (Figuras 1c, 1d e 1e).

De acordo com Silva O (2013), no contexto geral, a paciente apresentava características de oclusão próximas da normalidade para esta fase do desenvolvimento da oclusão, porém com um trespasse vertical aumentado, provavelmente já relacionado com o desgaste dentário vertical acentuado, que causa diminuição da altura facial anterior inferior da face (Figura 1). 
Figura 1 - 1a. Foto frontal da face; 1b. foto lateral da face; $1 \mathrm{c}$. Foto intrabucal lateral direita, 1d. Foto intrabucal frontal; 1e. Foto intrabucal lateral esquerda.

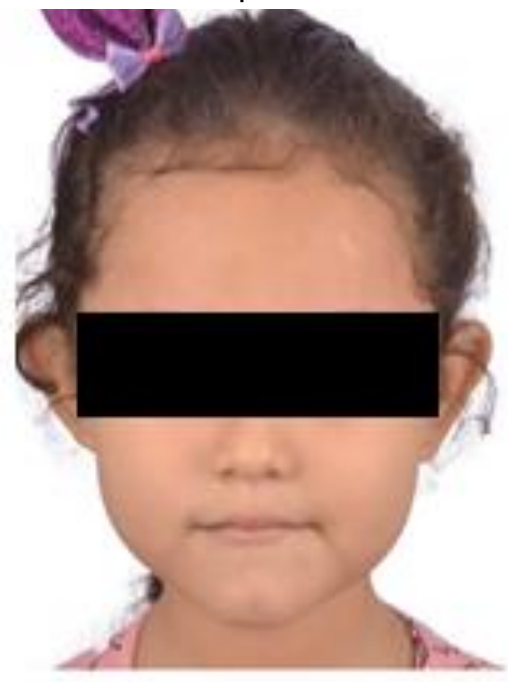

a

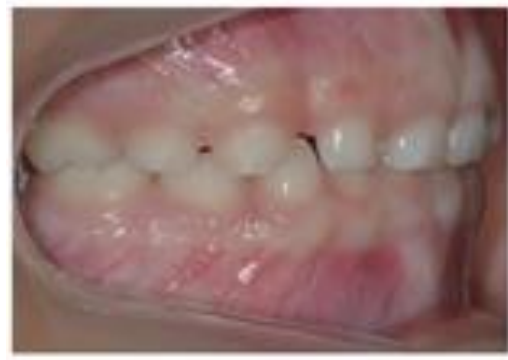

C

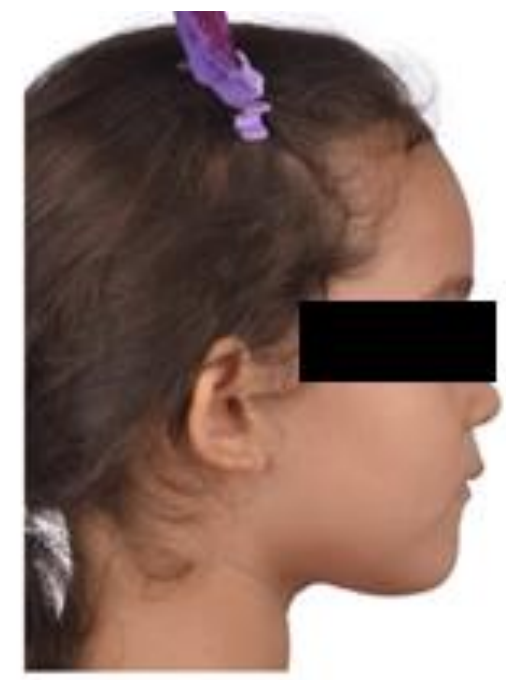

b

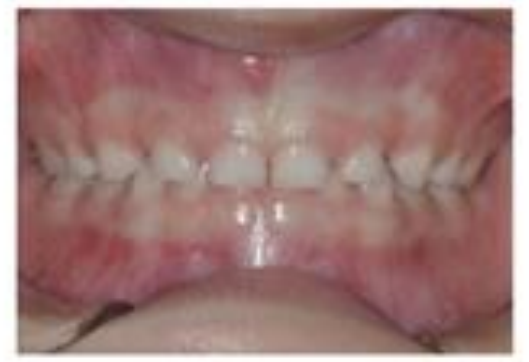

d

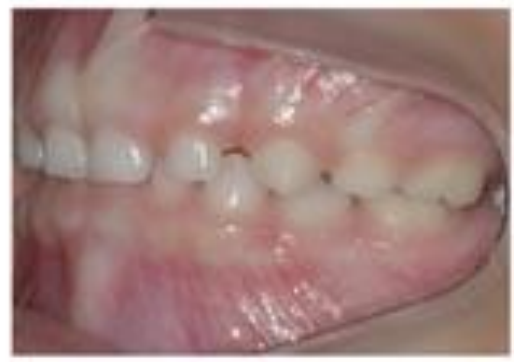

e

Fonte: Paiva PVO, et al., 2019.

$\mathrm{Na}$ análise radiográfica, verificou-se na radiografia panorâmica que a paciente apresentava características de normalidade para a dentadura decídua e ainda uma boa predição no que se diz respeito ao desenvolvimento para as dentaduras mista e permanente, pela presença de todos os dentes sucessores, com espaço suficiente para suas erupções (Figura 2).

Figura 2 - Radiografia panorâmica inicial.

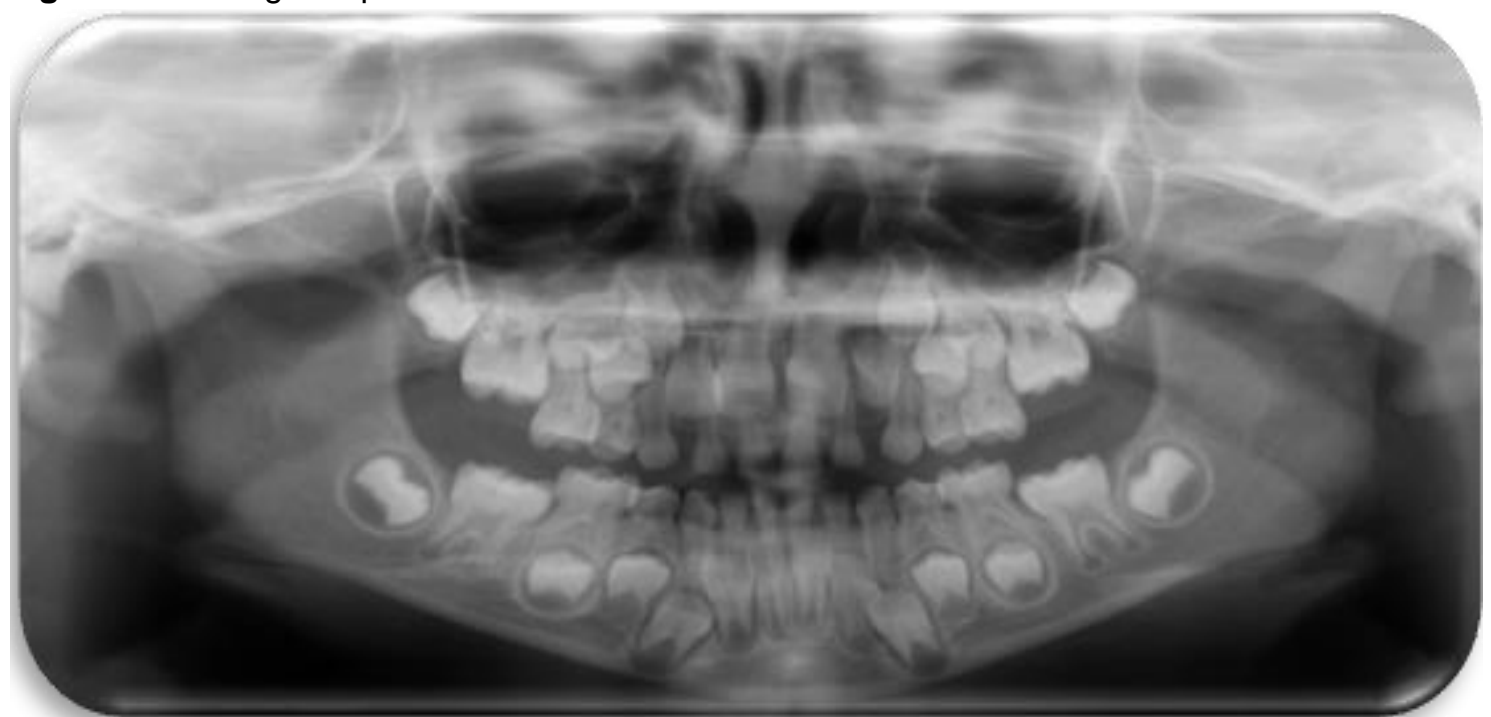

Fonte: Paiva PVO, et al., 2019. 
No intuito de detalhar a avaliação imaginológica da severidade do desgaste inicial, bem como realizar acompanhamento longitudinal, foi realizada uma radiografia periapical dos incisivos superiores e inferiores (Figura 3).

Figura 3 - 3a. Radiografia periapical inicial dos incisivos superiores; 3b. Radiografia periapical inicial dos incisivos inferiores.

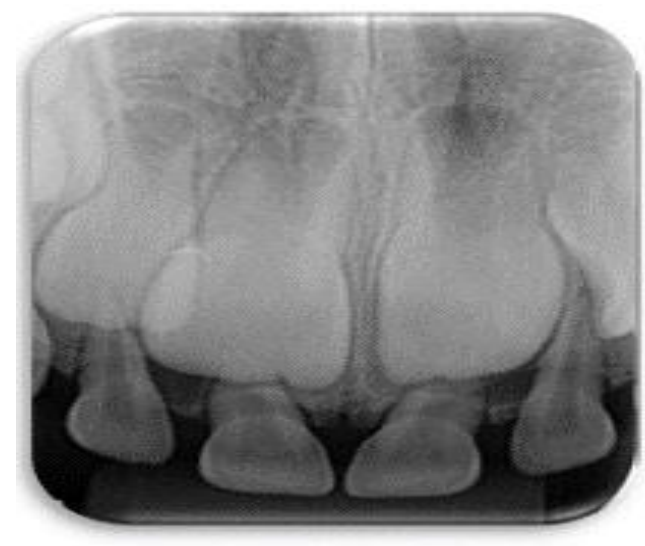

Fonte: Paiva PVO, et al., 2019.

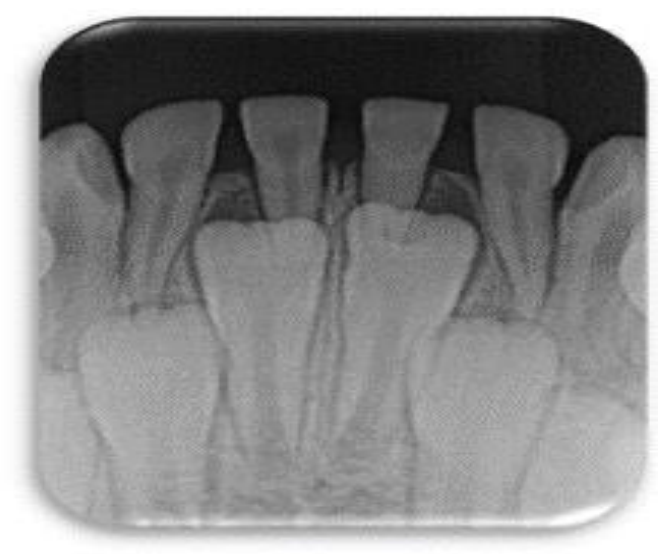

b

Na Figura 3, pode-se confirmar a imagem clínica de desgaste acentuado generalizado na região de incisivos, principalmente na região de incisivos inferiores.

Para complementar o diagnóstico, prognóstico e viabilizar o plano de tratamento, foi avaliada a telerradiografia em norma lateral, com o intuito de realizar a análise morfológica e numérica da tipologia e padrão de crescimento facial da paciente, tendo sido verificado que ela apresentava uma tipologia braquifacial, com medidas cefalométricas caraterísticas de um indivíduo padrão I (Figura 4).

Figura 4 - Telerradiografia em norma lateral inicial da paciente.

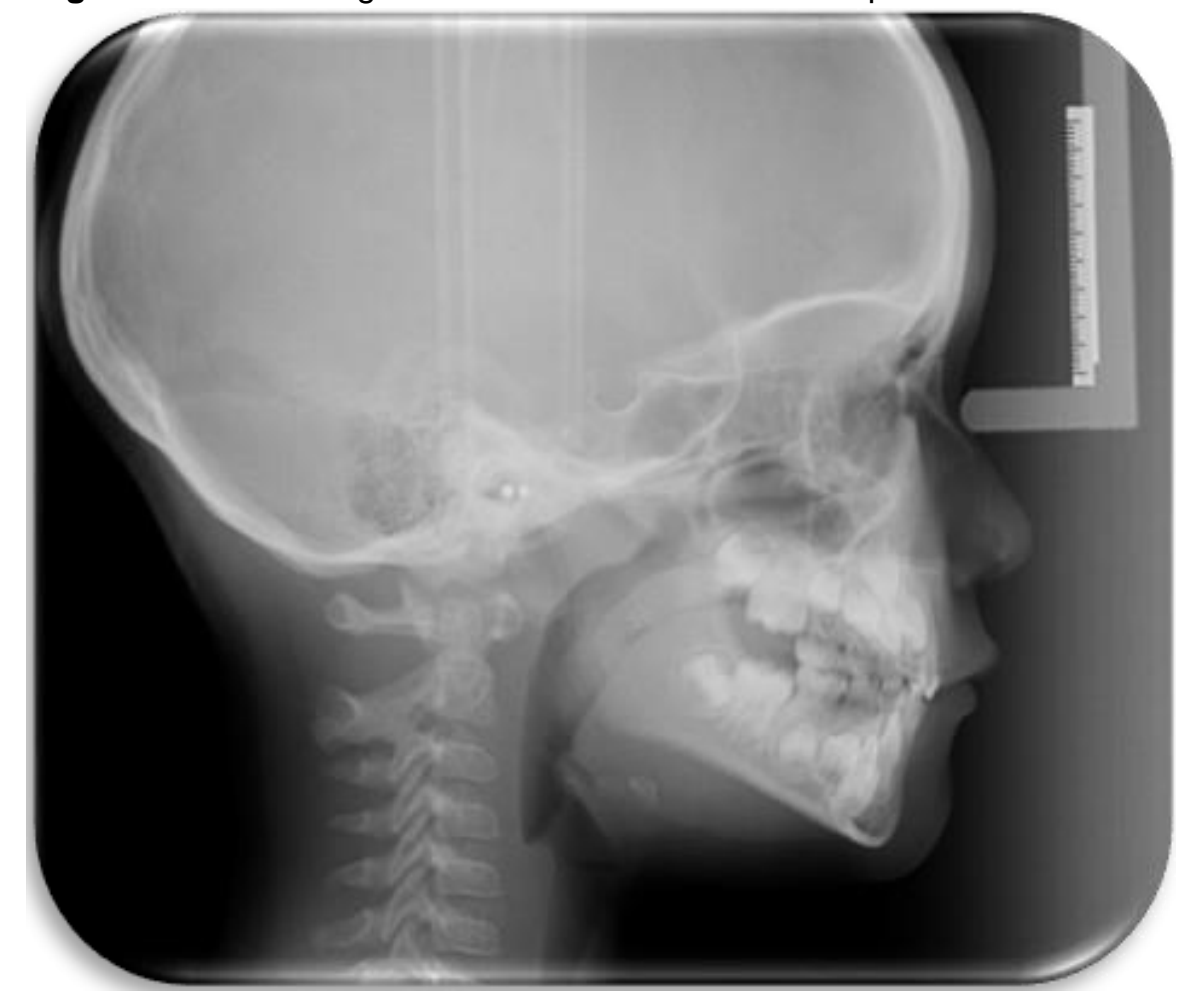

Fonte: Paiva PVO, et al., 2019. 
Com os dados clínicos e após a avaliação da documentação, a anamnese realizada e a ficha clínica preenchida, pôde-se desenvolver um plano de tratamento. Vale a pena ressaltar que, ao examinar clinicamente a articulação temporomandibular (ATM), não foram constatados estalido, dor, ruído ou dificuldade durante os movimentos de abertura e fechamento da boca.

A terapêutica escolhida no plano de tratamento foi a utilização da placa de mordida rígida de resina acrílica, funcionando como base de um tratamento interdisciplinar por envolver clínica odontológica infantil (Odontopediatria e Ortodontia), pediatria médica e psicologia.

Esse procedimento foi escolhido em função do claro diagnóstico relacionado ao bruxismo do sono, no qual a paciente apresentou: presença de episódios de bruxismo durante o sono, com a erupção dos incisivos centrais superiores e inferiores decíduos, sono leve e dificuldade de dormir. Um quadro de problema respiratório, relacionado à rinite alérgica, também foi diagnosticado.

A placa de mordida rígida foi utilizada com o objetivo de funcionar como um anteparo aos movimentos parafuncionais. A abordagem médica apresentou-se relacionada à resolução dos possíveis sinais e sintomas relacionados ao bruxismo do sono. Já o tratamento psicológico visou ao controle da ansiedade e autoconhecimento. Após o esclarecimento da criança e dos responsáveis quanto às possíveis causas do bruxismo do sono e quanto aos benefícios do tratamento, a paciente foi encaminhada para o tratamento psicológico.

Na sessão seguinte, realizou-se moldagem das arcadas superior e inferior com alginato em moldeiras ortodônticas, depois imediatamente vazou-se o gesso tipo pedra IV. É importante lembrar que uma etapa imprescindível para esta montagem dos modelos no ASA é o registro maxilo-mandibular para confecção da placa.

Assim, duas ou três lâminas de cera 7 foram recortadas, suavemente aquecidas e inseridas na cavidade bucal, para, inicialmente, tirar o registro da arcada superior e depois, com a manipulação da mandíbula, registrar a relação cuspídea entre as arcadas, seguindo-se imediatamente a refrigeração da lâmina de cera.

Assim, após aguardar o tempo de cristalização, os modelos foram recortados e montados em articulador semi-ajustável. A placa foi confeccionada, então, sobre o modelo superior utilizando-se resina acrílica (Figura 5).

Figura 5 - Placa da paciente confeccionada sobre o modelo superior.

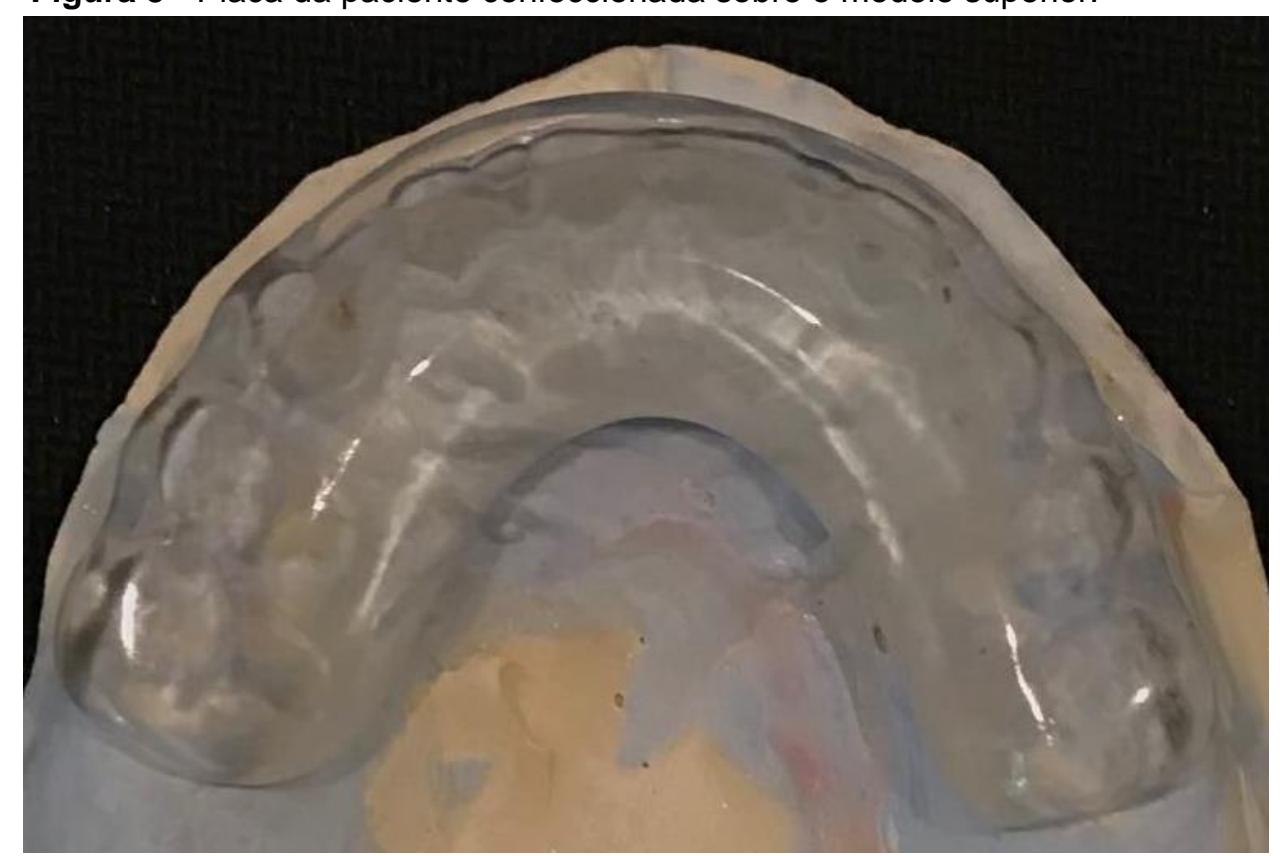

Fonte: Paiva PVO, et al., 2019. 
Foram considerados os seguintes critérios antes da instalação da placa: perfeito encaixe aos dentes, estabilidade e retenção, contatos de bordas incisais e pontas de cúspides antagonistas numa superfície plana, sem marcas ou planos inclinados, os dentes posteriores em contato com a placa com a mesma intensidade e os dentes anteriores com menos intensidade, durante os movimentos de lateralidade, haver apenas contato dos caninos, e nos movimentos de protrusão haver o contato dos incisivos inferiores, desde que com menor intensidade, e, finalmente, ter em vista que a placa deverá estar polida para não irritar os tecidos moles (VALLE RT, et al., 2015). A placa foi instalada seguida dos ajustes oclusais realizados diretamente na boca da paciente (Figura 6).

Figura 6 - Visão intrabucal frontal da paciente utilizando a placa no momento da demarcação dos pontos de contato com a pinça cruzada e o papel carbono.

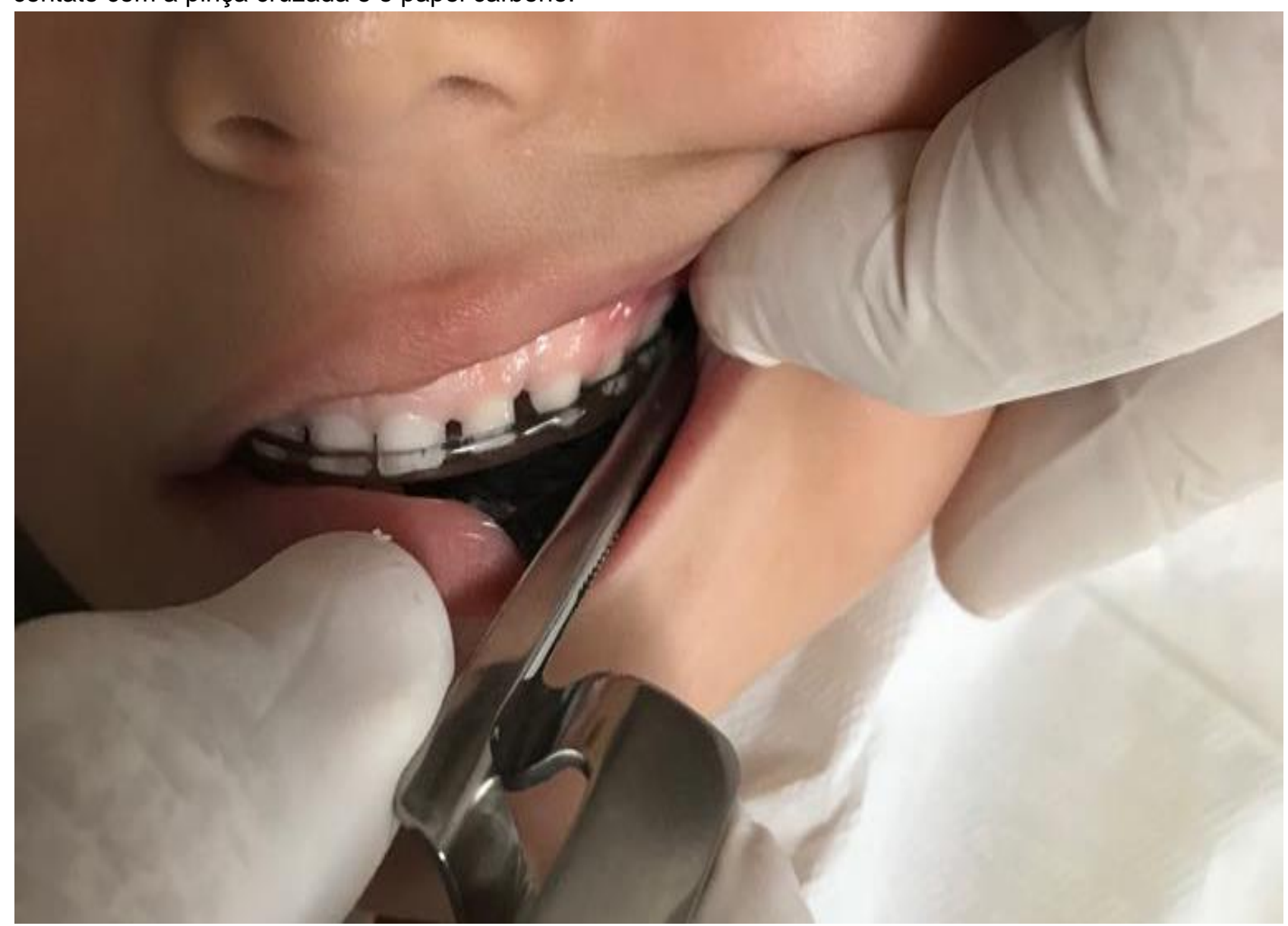

Fonte: Paiva PVO, et al., 2019.

Os responsáveis e a criança foram orientados quanto aos cuidados e período de utilização da placa, sendo este determinado para o uso noturno, uma vez que a paciente realizava o ranger de dentes no período da noite, enquanto dormia.

Os retornos para controle e avaliação ocorreram com a seguinte estratificação: mensal, bimensal e trimestral. Realizou-se sempre a demarcação dos pontos e estudo da presença de desgastes e até mesmo de fraturas na placa, bem como foram feitos os ajustes da placa para remoção de irregularidades para evitar o surgimento de vícios de postura.

Após um ano de uso, observou-se: a estabilização dos desgastes na região dos incisivos superiores e inferiores. E há ainda o relato dos responsáveis sobre a diminuição progressiva e, subsequentemente, o desaparecimento do bruxismo noturno apresentado pela paciente antes do início do tratamento, atestandose o sucesso deste. 


\section{DISCUSSÃO}

A principal ação clínica associada ao bruxismo deve ser voltada aos cuidados de proteção aos dentes, abrandando o ranger, diminuindo dores faciais e temporais e proporcionando melhorias na qualidade do sono.

Há três linhas de estratégias que devem ser aplicadas: dentária, psico-comportamental e farmacológica (BRIGUENTE GL, 2017). Conforme Souza KM (2010), A incidência do bruxismo pode variar entre $5 \%$ a $81 \%$ em crianças, estando comparável a $15 \%$ a $88 \%$ reportados em adultos e de ocorrência em população geral em $90 \%$.

Como a etiologia do bruxismo é geralmente uma associação de fatores, o tratamento psicológico é de fundamental importância, quando iniciada com o uso da placa de mordida. Essa placa reduz a atividade parafuncional, desprogramar e induzir o relaxamento muscular, para obter uma proteção dos dentes reduzindo assim o desgaste, atrição, balanceio dos contatos oclusais, assim como o reposicionamento da mandíbula, colocando-a em uma relação balanceada com a maxila para que se possa alcançar um equilíbrio neuromuscular.

Além disso existe um sistema inteligente que utiliza uma "terapia de biofeedback" que consiste em uma placa com estímulos elétricos através de um chip por transmissão via bluetooth que balanceia forças oclusais maxilomandibular (GAO J, et al., 2020).

A abordagem oclusal é subdividida em "reversível" e "irreversível". A subdivisão "irreversível" abrange métodos de ajustes seletivos oclusais permanentes, restaurações dentárias e protéticas e ainda tratamento ortodôntico.

Devido à alteração do paradigma etiológico do bruxismo, que passou de uma etiologia que assentava na mecânica oclusal para uma visão de origem e controle central, estas alterações oclusais não estão, na maioria dos casos, indicadas.

São raramente aplicadas e, nesses casos, a sua aplicação deve-se a suspeitas de que a etiologia do bruxismo está relacionada com fatores oclusais. Essa abordagem oclusal poderá minimizar danos causados pelo ranger de dentes, mas não eliminará o bruxismo (CASTRILLON EE, 2016).

Por outro lado, a abordagem oclusal reversível é o método mais utilizado pelos clínicos. Esse método consiste na utilização de aparelhos intraorais removíveis colocados entre os maxilares. As placas oclusais podem ser confeccionadas em diferentes materiais, podendo ser rígidas ou resilientes, e podem diferir quanto à sua espessura e extensão da cobertura oclusal, estabelecendo diferentes relações intermaxilares consoante a sua indicação terapêutica (DIAS RAB, 2015).

Segundo Lee SJ (2010), o bruxismo é uma patologia com origem no sistema nervoso central, com consequências odontológicas, não sendo, portanto, o cirurgião-dentista, isoladamente, que garantirá o tratamento mais adequado para esse quadro patológico.

Mesmo que temporariamente, pode-se afirmar que a toxina botulínica A pode atenuar os níveis de dor, porém não interfere na frequência dos eventos de bruxismo, apresentando-se como uma alternativa terapêutica conversadora para pacientes portadores dessa patologia. Assim, o tratamento com toxina botulínica A é um tratamento seguro e eficaz para esses pacientes, quando aplicado de maneira apropriada.

O ideal para o tratamento do bruxismo é a atuação multidisciplinar entre dentista, psicólogo e o próprio paciente motivado. Sendo assim, é de suma importância a proservação do paciente (PIZZOL KEDC, 2013). Para crianças que possuem bruxismo, o tratamento indicado deve ser conservador e reversível visando a não interferência negativa no padrão de normalidade de crescimento e desenvolvimento infantil.

Portanto, o cirurgião dentista deve estar sempre atento aos sinais e sintomas que pacientes possam vir a apresentar em seu consultório. Assim, este artigo procurou cumprir o objetivo de apresentar, por meio de um relato de caso, os fatores etiológicos, as consequências e os possíveis tratamentos para o bruxismo. 


\section{REFERÊNCIAS}

1. ANTONIO AG, et al. Bruxism in children: a warning sign for 41 psychological problems. Journal-Canadian Dental Association, 2006; 72(2): 155.

2. BRIGUENTE GL. Placa oclusal como opção de controle para o bruxismo do sono: revisão de literatura. Trabalho de conclusão de curso (bacharel em odontologia) - Universidade do Sul de Santa Catarina, Tubarão, 2017; 43 p.

3. CARIOLA CT. O desenho da figura humana de crianças com bruxismo. 2006;55(124):37-52.

4. CARRA MC. Sleep bruxism and sleep disorders in adolescents. Journal of Dentofacial Anomalies and Orthodontics, 2018, 21(1): 108.

5. CASTRILLON EE, et al. Sleep bruxism: an updated review of an old problem. Acta Odontologica Scandinavica, 2016; 74(5):328-334.

6. DEMIR A, et al. The relationship between bruxism and occlusal factors among seven- to 19-year-old Turkish children. Angle Orthod, 2004;74 (5):672-6.

7. DIAS RAB. Desenvolvimento de técnica laboratorial e avaliação clínica de goteiras oclusais rígidas obtidas por técnica assistida por computador (CAD/CAM) no tratamento sintomático/ortopédico de pacientes com diagnóstico de Bruxismo e/ou Disfunção Temporomandibular. Tese (doutorado em ciências da saúde) - Universidade de Coimbra, Coimbra, 2015.

8. DINIZ MB, et al. Bruxismo na infância: Um sinal de alerta para odontopediatras e pediatras. Revista Paulista de Pediatria, 2009; 27(3): 329-334.

9. GAO J, et al. Intelligent Occlusion Stabilization Splint with Stress-Sensor System for Bruxism Diagnosis and Treatment. Sensors, 2020; 20(1): 89.

10. GAMA EAOA, RIVA MC. Bruxismo: Uma revisão da literatura. Ciência Atual-Revista Científica Multidisciplinar das Faculdades São José, 2013; 1(1):16-97.

11. GIMENES MCM. Bruxismo aspectos clínicos e tratamentos. Disponível em:

12. http://WWW.portaleducação.com. br/odontologia/artigos/2008/bruxismo aspectos clínicos. Acesso em: 22/04/2012.

13. GOMES MAL, BARRETO SR. Bruxismo infantil: importância do controle dos fatores etiológicos e do diagnóstico na prevenção das desordens temporomandibulares em crianças-uma revisão de literatura ilustrada (UNIT-SE). Trabalho de conclusão de curso (bacharel em odontologia) - Universidade de Titradentes, Aracaju, Sergipe, 2019.

14. GONÇALVES LPV, et al. Relação entre bruxismo, fatores oclusais e hábitos bucais. Dental Press J. Othod, 2010; 15(2): 97104.

15. HACHMANN A, et al. Efficacy of the nocturnal bite plate in the control of bruxism for 3 to 5 year old children. J Clin Pediatr Dent 1999; 24:9-15.

16. JAIN A, et al. Bruxism: an obscure pain. Int J Dent Med Res, 2014, 1(1): 21-30.

17. KANATHILA H, et al. Diagnosis and treatment of bruxism: Concepts from past to present. Int. J. Appl. Dent. Sci, 2018; 4(1): 290-5.

18. KARIMI M. Bruxism in children: causes and solutions. J Dent Health Oral Disord Ther, 2018; 9(2): 150-152.

19. KATO T, LAVIGNE GJ. Sleep bruxism: a sleep-related movement disorder. Sleep Medicine Clinics, $2010 ; 5(1): 9-35$.

20. KUCH EV, et al. Bruxing and non-bruxing children: a comparison of their personality traits. Pediatr Dent, $1979 ; 1(3): 182-7$.

21. LEE SJ, et al. Effect of botulinum toxin injection on nocturnal bruxism: a randomized controlled trial. American journal of physical medicine \& rehabilitation, 2010; 89(1): 16-23.

22. LOBBEZOO F. Principles for the management of bruxism. J Oral Rehabil, 2008; 35(7):509-23.

23. LOBBEZOO F, et al. Bruxism defined and graded: an international consensus. $J$ Oral Rehabil 2013;40(1):2-4

24. MACHADO E, et al. Prevalence of sleep bruxism in children: a systematic review. Dental press journal of orthodontics, 2014 , 19(6): 54-61.

25. MACHADO E, et al. Bruxismo do sono: possibilidades terapêuticas baseadas em evidências. Dental Press Journal of Orthodontics, 2011;16(2): 58-64.

26. MACIEL RN. Bruxismo. São Paulo: Artes Médicas; 2010.

27. MALTAGLIATI LA, MONTES LAP. Análise dos fatores que motivam os pacientes adultos a buscarem o tratamento ortodôntico. Revista Dental Press de Ortodontia e Ortopedia Facial, 2007; 12(6): 54-60.

28. OLIVEIRA ME, CARMO MRC. Placa de mordida interoclusal para tratamento de bruxismo. Revista do CROMG, 2000;7(3):183-186.

29. OLIVEIRA MT, et al. Bruxism in children: Effectiveness of bite splints. Journal of Research in Dentistry, 2014, 2(1): 22-29.

30. PACHECO MCT, et al. Guidelines proposal for clinical recognition of mouth breathing children. Dental press journal of orthodontics, 2015; 20(4):39-44.

31. PAESANI DA. Bruxism: theory and practice. New Malden, UK: Quintessence Publishing; 2010.

32. PIZZOL KEDC, et al. Bruxismo na infância: fatores etiológicos e possíveis tratamentos. Revista de Odontologia da UNESP, 2013; 32(5): 157-163.

33. PRADO IM, et al. Sleep bruxism and orthodontic appliance among children and adolescents: a preliminary study. J Sleep Disord Ther, 2016, 5(2): 1-7.

34. REAL APARICIO MC. Disfunción temporomandibular: causas y tratamientos. Rev. Nac. (Itauguá), Itauguá , 2008; 10(1): 68-91.

35. RESTREPO CC, et al. Effect of occlusal splints on the temporomandibular disorders, dental wear and anxiety of bruxist children. European journal of dentistry, 2011; 5(04): 441-450.

36. RIOS LT, et al. Bruxismo infantil e sua associação com fatores psicológicos-revisão sistemática da literatura. Revista de Odontologia da Universidade Cidade de São Paulo, 2018;30(1):64-76.

37. SAMPAIO NM, et al. Relationship between stress and sleep bruxism in children and their mothers: A case control study. Sleep Science, 2018;11(4): 239, 2018.

38. SILVA O, et al. Ortodontia interceptiva: protocolo de tratamento em duas fases. São Paulo: Artes Médicas; $2013: 574$.

39. SOUZA KM, et al. Bruxismo infantil: prevalência, etiologia, diagnóstico e tratamento uma abordagem literária:[revisão]. Ortho Sci., Orthod. sci. pract, 2010; 39(10): 145-149.

40. VALLE RT, et al. Disfunções Temporomandibulares: Abordagem Clínica. Nova Odessa - SP: Napoleão Quintessence; 2015.

41. VANDERAS AP, et al. Úrinary catecholamine levels and bruxism in children. J Oral Rehabil. 1999; 26(2):103-10. 